\title{
Final performance and lesson-learned of SAXO, the VLT-SPHERE extreme AO From early design to on-sky results
}

\author{
T. Fusco*a,b, J.-F. Sauvage ${ }^{\mathrm{a}, \mathrm{b}}$, C. Petit ${ }^{\mathrm{a}}$, A. Costille ${ }^{\mathrm{b}}$, K. Dohlen ${ }^{\mathrm{b}}$, D. Mouillet ${ }^{\mathrm{c}}$, J-L Beuzit $^{\mathrm{c}}$ \\ M. Kasper ${ }^{\mathrm{d}}$, M. Suarez ${ }^{\mathrm{d}}$, C. Soenke ${ }^{\mathrm{d}}$, E. Fedrigo ${ }^{\mathrm{e}}$, M. Downing ${ }^{\mathrm{c}}$, P. Baudoz ${ }^{\mathrm{e}}$, A. Sevin ${ }^{\mathrm{e}}$, \\ D. Perret ${ }^{\mathrm{e}}$, A. Baruffolo ${ }^{\mathrm{f}}$, B. Salasnich ${ }^{\mathrm{f}}$, P. Puget ${ }^{\mathrm{c}}$, F. Feautrier ${ }^{\mathrm{c}}$, S. Rochat ${ }^{\mathrm{c}}, \mathrm{T}^{\mathrm{T}}$. Moulin ${ }^{\mathrm{c}}$, A. \\ Deboulbéc, E. Hugot $^{\mathrm{b}}$, A. Vigan ${ }^{\mathrm{b}}$, D. Mawet ${ }^{\mathrm{d}}$, J. Girard ${ }^{\mathrm{d}}$, N. Hubin ${ }^{\mathrm{d}}$ \\ aONERA, 29 avenue de la Division Leclerc, 92322 Châtillon, France \\ ${ }^{\mathrm{b}}$ Aix Marseille Université, CNRS, LAM UMR 7326, 13388, Marseille, France \\ 'IPAG (UMR 5274) BP 53. F-38041 Grenoble, France

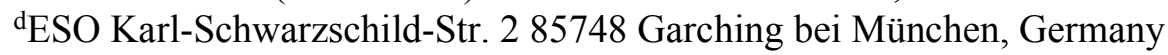 \\ 'LESIA Observatoire de Paris, 5, place Jules Janssen 92195 Meudon, France \\ fINAF Osservatorio Astronomico di Bologna ,Via Ranzani 1, 40127, Bologna, Italy
}

\begin{abstract}
The extreme AO system, SAXO (SPHERE AO for eXoplanet Observation), is the heart of the SPHERE system, feeding the scientific instruments with flat wave front corrected from all the atmospheric turbulence and internal defects. We will present the final performance of SAXO obtained during the instrument AIT in Europe as well as the very first on-sky results. The main requirements and system characteristics will be recalled and the full AO loop performance will be quantified and compared to original specifications. It will be demonstrated that SAXO meets or even exceeds (especially its limit magnitude and its jitter residuals) its challenging requirements (more than $90 \%$ of SR in $\mathrm{H}$ band and a 3 mas residual jitter). Finally, after 10 years of AO developments, from early design to final on-sky implementations, some critical system aspects as well as some important lesson-learned will be presented in the perspective of the future generation of complex AO systems for VLTs and ELTs.
\end{abstract}

Keywords: Key words. Instrumentation: Adaptive Optics, Coronagraph, High Contrast Imaging - Methods: Wavefront Sensing, Control, Phase Diversity, Shack-Hartmann

\section{INTRODUCTION}

Direct detection and spectral characterization of exoplanets is one of the most exciting but also one of the most challenging areas in the current astronomy. In that framework, the SPHERE (Spectro-Polarimetry High-contrast Exoplanet Research) instrument has been recently installed on UT3, one of the four 8-m telescopes of the European Southern Observatory Very Large Telescope (ESO VLT) at Paranal (Chile). The main scientific objective of SPHERE [1] is the direct detection of photons coming from giant extrasolar planets (between 1 and 20 Jupiter masses). Any detection will then be followed by a first characterization of the planet atmosphere (clouds, dust content, methane, water absorption...). In addition, the survey of an extended number of stars (typically a few hundreds) is mandatory for performing meaningful statistical studies. Such extremely challenging scientific objectives directly translate into a relatively complex high-contrast instrument. Coronagraphic and smart imaging capabilities are essential for reaching the high contrast (close to the optical axis) required for direct extrasolar planet detection. From the ground, the core of any high-contrast instrument is an extreme adaptive optics (XAO) system. Such a system must be capable of making corrections for the perturbations induced by the atmospheric turbulence as well as for the internal aberrations of the instrument itself. In that context, SAXO (Sphere Ao for eXoplanet Observation), the XAO system of SPHERE has been designed, integrated, tested in labs and finally on sky, by a European wide group of AO scientists and Engineer, led by ONERA with the strong support of ESO. A comprehensive design of SAXO and the detailed description of the main system choices can be found in [2]. We report here the final AIT (Assembling Integration and Test) of SAXO which

Adaptive Optics Systems IV, edited by Enrico Marchetti, Laird M. Close, Jean-Pierre Véran, Proc. of SPIE Vol. 9148, 91481U · (c) 2014 SPIE CCC code: $0277-786 X / 14 / \$ 18 \cdot$ doi: $10.1117 / 12.2055423$ 
took place in Grenoble from 2012 to 2013, as well as the very first on-sky results, obtained in May 2014 on UT3, one of the four eight meter class telescope of the VLT.

A detailed description of SPHERE overall performance will be found in [4]

\section{SAXO DESCRIPTION}

\subsection{SPHERE overview}

The SPHERE system aims at detecting extremely faint sources (giant extrasolar planets) in the vicinity of bright stars. Such a challenging goal requires the use of a very-high-order performance AO system, a coronagraphic device to cancel out the flux coming from the star itself, and smart focal plane techniques to calibrate any coronagraph imperfections and residual uncorrected turbulent or static wavefronts. The detection limit for the SPHERE instrument is 10-6 (i.e,15 magnitudes between star and the planet) with a goal around 10-8. There is no direct link between the AO system performance and the final detectivity of the instrument; nevertheless, the impact of $\mathrm{AO}$ on the final performance is related to the performance of the coronagraph. A better AO correction leads to a better coronagraph extinction and therefore leads to the following improvements in system performance:

- a reduction of the photon and flat-field noises (i.e., a gain in Signal-to-Noise Ratio for a given integration time)

- a reduction of the static speckle (through the reduction of airy pattern intensity due to the coronagraph optimization).

These reductions are important from the global system performance point of view, and the optimization of the coronagraph rejection is a main goal of the SPHERE system. It of course requires the use and the optimization of an $\mathrm{XAO}$ system, as presented in the following. Nevertheless, the ultimate detection limit will be achieved through an extreme control of system internal defects (non-common path aberrations (NCPAs), optical axis decentering, vibrations, coronagraph and imaging system imperfections, and so on). This ultimate control will also be partially ensured by the AO system through the use of additional devices in the AO concept To meet the requirements (and hopefully the goal) in terms of detection (AD3) the proposed design of SPHERE (see AD1) is divided into four subsystems, namely, the common path optics and three science channels. The common path includes pupil-stabilizing fore-optics (tip-tilt and derotator) where insertable polarimetric half-wave plates are also provided, the SAXO XAO system with a visible wavefront sensor, and near infrared (NIR) coronagraphic devices in order to feed the infrared dual-imaging spectrograph (IRDIS) and the integral field spectrograph (IFS) with a highly stable coronagraphic image in the NIR. The three scientific channels gather complementary instrumentation to maximize the probability of exoplanet detection and to give us access to a large range of wavelengths and information (e.g., imaging, spectra, and polarization).

The concept behind this very challenging instrument is illustrated in Figure 1, where the common NIR-Vis beam is indicated in orange, the exclusively NIR beam is indicated in red, and the exclusively Vis beam is indicated in blue.

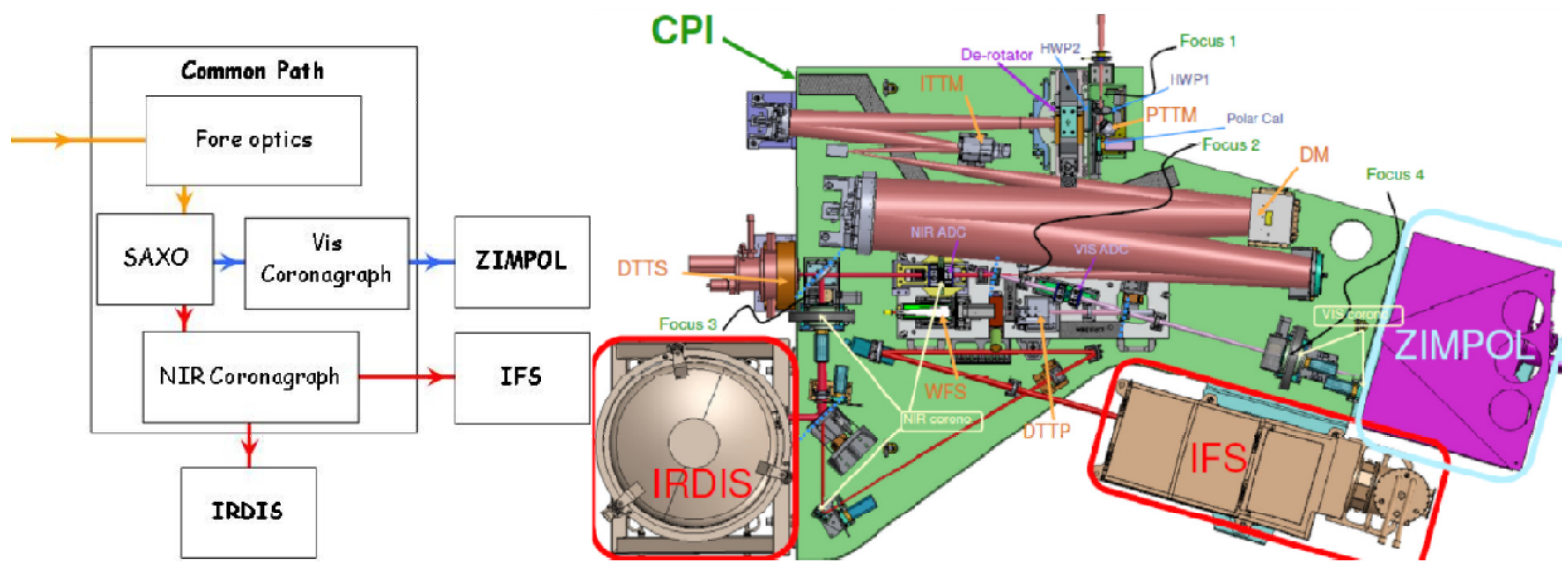

Figure 1 Global concept of the SPHERE instrument, indicating the four subsystems and the main functionalities within the common path subsystem. Optical beams are indicated in red for NIR, blue for Vis, and orange for common path 

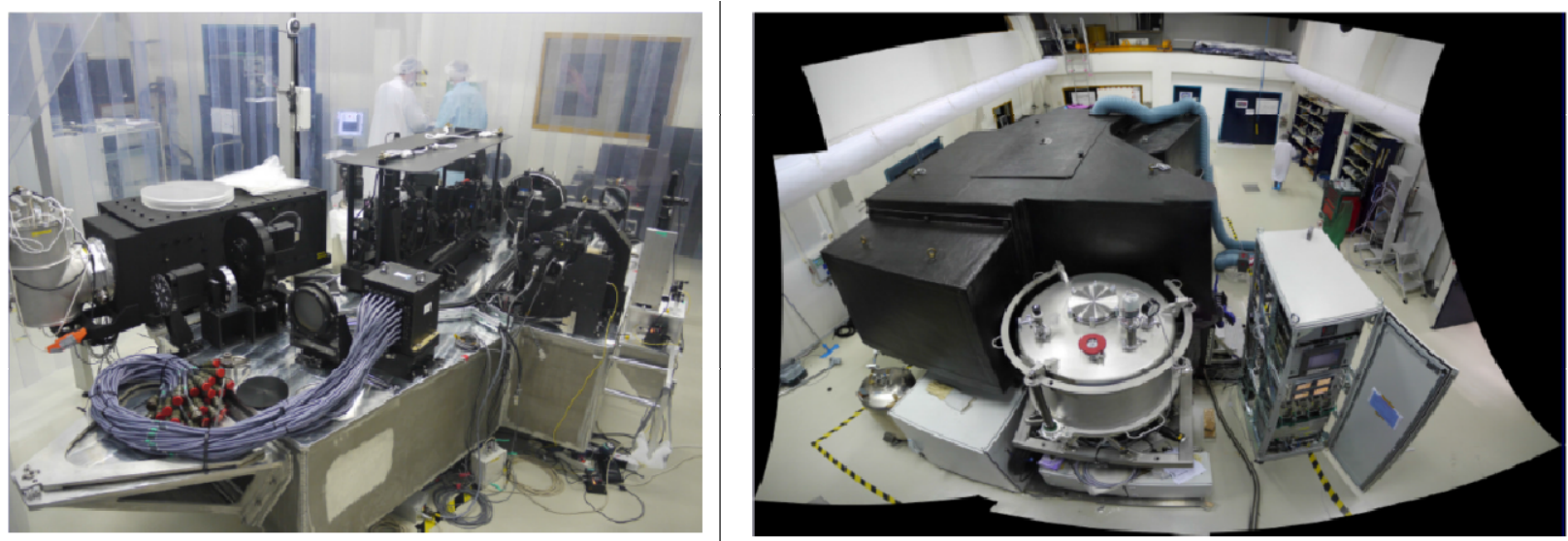

Figure 2 [Left] SAXO being integrated in the CPI and [Right] SPHERE in its final shape at IPAG (8 tons, $6 \mathrm{~m}$ length, $4 \mathrm{~m}$ large, $2.5 \mathrm{~m}$ height)

\subsection{The SAXO requirements}

The extreme AO system (SAXO) is the core of the SPHERE instrument, and is essential for reaching the extremely high contrast requirements. In this framework, the SAXO must fulfill the following three high-level requirements (see [2]):

- Ensure the measurement and correction of the turbulent phase perturbations of the telescope and system common optics aberrations and of the NCPAs (main AO loop);

- Ensure an extremely high stability (at low temporal frequency) of the optical axis at the level of the coronagraphic mask (using a IR sensor at the level of the coronagraphic mask, the Differential Tip Tilt Sensor [DTTS])

- $\quad$ Ensure the measurement and the correction of any pupil motion (using a pupil motion sensor [PMS]).

\subsection{SAXO design and implementation}

The SAXO system will have to compensate for any wave front aberrations and optical misalignment. The driving goal for the scientific instrument is very high contrast imaging in the near IR. A general overview of SAXO is given in Figure 3

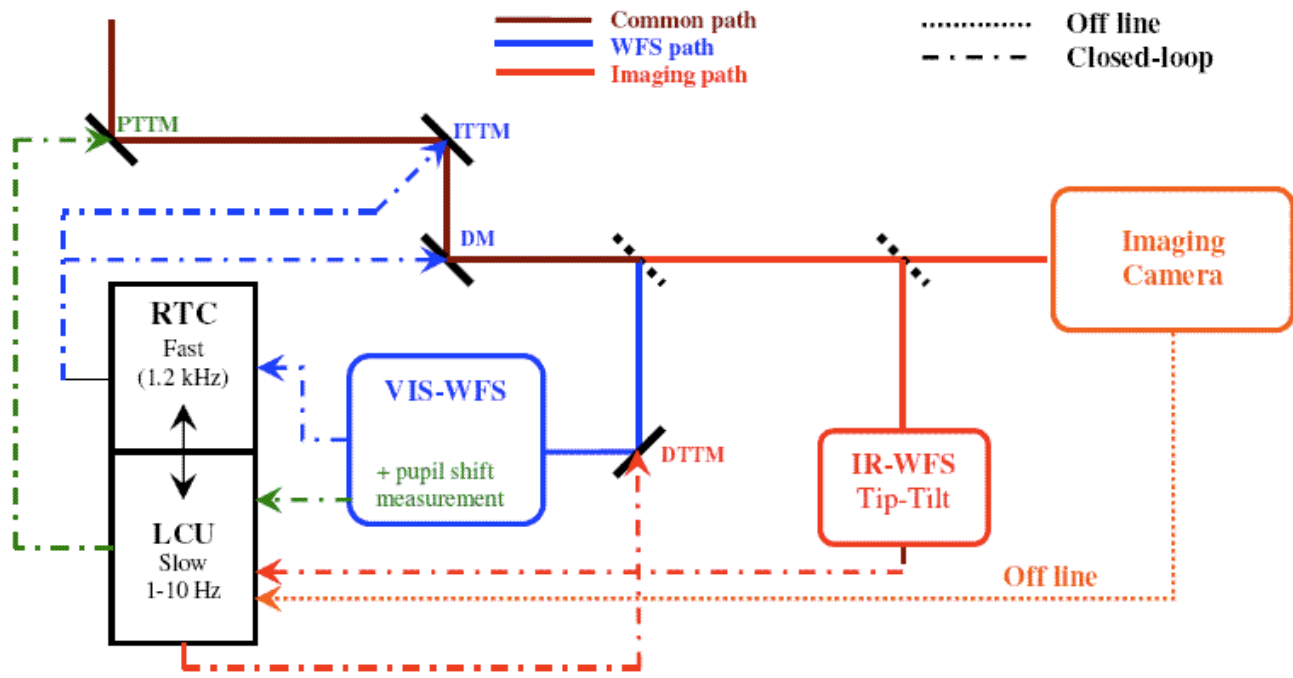

Figure 3 Schematic representation of SAXO loops 
The SAXO system is composed by 3 loops plus an off line one for calibration purpose.

- Main AO loop (1.2 kHz): correct for atmospheric, telescope and common path defects. The impact is the increase of detection signal to noise ratio through the reduction of the smooth PSF halo due to turbulence effects

- The DTT loop for fine centering on coronagraph mask (correction of differential tip-tilt between VIS and IR channel). It will ensure an optimal performance of the coronagraph device

- The PTT loop for pupil shift correction (telescope and instrument). It will ensure that the uncorrected instrumental aberrations effects (in the focal plane) will always be located at the same position and thus will be canceled out by a clever post-processing procedure

- NCPA pre-compensation which will lead to the reduction of persistent speckle

In addition to these loops, the AO system will also provide:

- $\quad$ anti-wind up + Garbage Collector loop allowing to deal with saturated actuators

- a Tip Tilt and focus off-load process on telescope M2

- a turbulence parameter and system performance on-line monitor

To sense any relevant perturbation and correct for it, SAXO gathers the following elements:

- A high spatial and temporal frequencies deformable mirror to correct for phase perturbations but the tip-tilt. The 41x41 actuators DM has been provided by CILAS Company. This component does not fulfill its specification in terms of dead actuators (17 are declared "dead" in the SAXO system at the moment this paper is written) and its shape at rest which evolves with $\mathrm{T}^{\circ}$ (typically $1 \mu \mathrm{m}$ per degree and can represent up to $100 \%$ of the DM stroke itself for $\mathrm{T}^{\circ}$ exceeding $18^{\circ} \mathrm{C}$ ). The DM is now identified as the main risk for the SPEHRE system. Section 4 gives an overview of the DM status

- A fast image TTM (ITTM), provided by LESIA (Observatoire de Paris) and located in a pupil plane for image motion correction. This component does not fulfill its specification (phase lag of $13^{\circ}$ and $11^{\circ}$ for each axis instead of $5^{\circ}$ ). Nevertheless, this discrepancy has been considered as acceptable by the SAXO team and the component has been formally accepted

- A 40x40 visible spatially filtered Shack-Hartmann (VIS-WFS). It includes an EMCCD provided by E2V with the following characteristics $240 \times 240$ pixels (pixel size is $24 \times 24 \mu \mathrm{m}^{2}$ ). The final detector has been provided by ESO in july 2013. This component fulfills (or even exceeds) its specifications in terms of noise $(<0.1 \mathrm{e}-$ ), readout speed $(>1300 \mathrm{~Hz}$ ), quantum efficiency and Pixel PSF. In particular, these values contribute to the exceptional performance of the system in terms of limit magnitude (see section 8)

- A weighted Centre of Gravity (WCOG) algorithm shall be considered for slope estimation. This algorithm have been successfully implemented and tested in SPARTA.

- A spatial filter device is added in front of the WFS to reduce the aliasing effects. The filter shape is square with a variable size (from 0.7 to 2.1 arcsec). This component fulfills the specification in terms of speed and accuracy of size variations as well as centering w.r.t. optical axis (30 mas on sky)

- $\quad$ Real Time Computer (SPARTA) provided by ESO completely fulfill all the specification defined in RD1 and RD2. In particular, it exceeds its goal in terms of RTC latency since a $80 \mu$ s has been measured (spec was 150 $\mu$ s with a $100 \mu$ s goal). CCD read out + CCD controller delay + RTC latency + DM high voltage amplifier leads to a 2.14 frame overall delay at $1200 \mathrm{~Hz}$ for the main SAXO loop which exceed the specifications. The RTC includes a mixed control law: an Optimal Modal Gain Integrator for the DM mode and a Kalman filter base control law for the tip-tilt mode (Linear Quadratic Gaussian (LQG) control). This Kalman filter control law can correct for 10 vibration patterns located above the AO system bandwidth

- A phase diversity algorithm is used to measure and optimize the non-common path aberrations (NCPA). The algorithm has been successfully implemented in INS and the residual NCPA after the iterative compensation process completely fulfill the specification (measured SR is larger than $99 \%$ in $\mathrm{H}$ band which means less than $20 \mathrm{~nm}$ rms of resdiuals and less than $5 \mathrm{~nm}$ on the 50 first modes).

- A slow pupil TTM (PTTM) close to the entrance focal plane to correct for pupil shifts. The PTTM loop (which directly uses the WFS intensity) has been successfully implemented in SAXO and it has been shown that the loop is able to stabilize the SPHERE pupil with accuracy largely better than the $0.5 \%$ of the full pupil.

- A slow infra-red tip-tilt sensor (DTTS) on the scientific channel measuring the differential tip-tilt between the common and imaging paths has been successfully implemented. The DTTS loop characteristics fulfils the specification (in term of measurement accuracy and loop bandwidth) and the final accuracy in terms long term of optical axis stabilisation is lower than 0.5 mas which is well within the specifications. 


\section{AO SYSTEM ULTIMTE PERFORMANCE IN LABS}

\subsection{Ultimate performance on internal source}

Let us first consider the ultimate performance of the SPHERE system itself. The source is put at the entrance focal plane: FP1. SAXO loops are closed, Non-Common Path Aberration (NCAP) have been measured using a Phase diversity algorithm and pre-compensated for by modifying the WFS reference slopes [3].

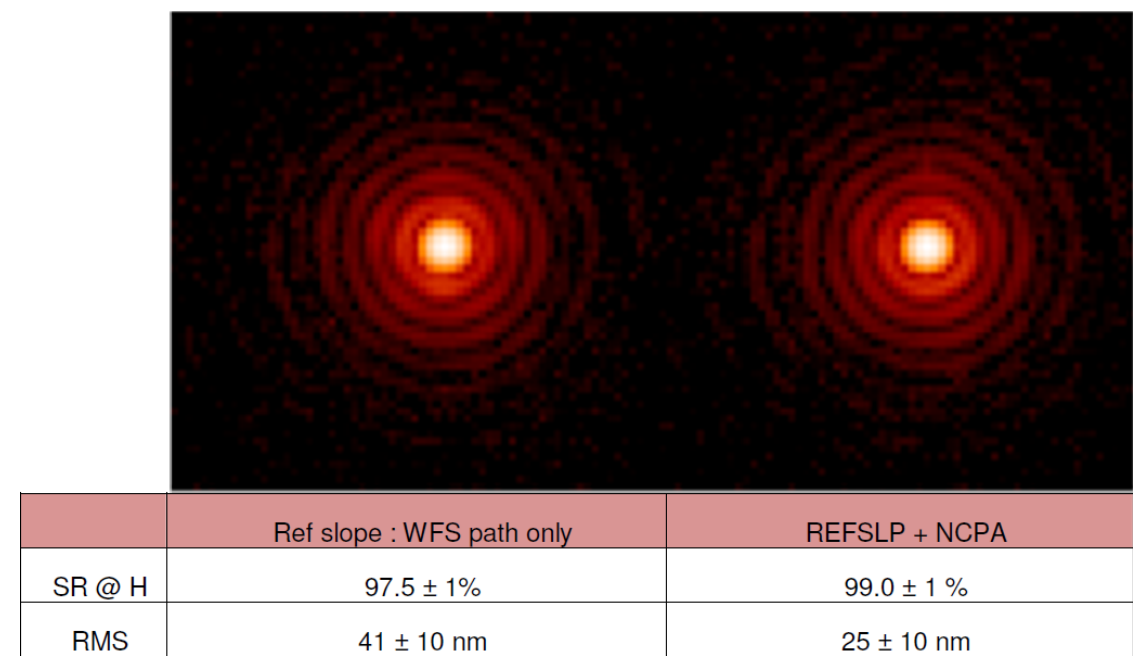

Figure 4 SPHERE internal performance without and with NCPA compensation.. This was obtained during AIT period with a limited number of dead actuator ( 4 only). $99 \%$ of SR in $\mathrm{H}$ (or in other words less than $25 \mathrm{~nm}$ rms of residual aberrations) have been obtained at the very end.

Considering only the correction of the WFS path aberrations (by using a internal fiber at the entrance of the WFS arms), the SPHERE optical quality is already impressive with a SR in H around $97.5 \%$ which corresponds to $41 \mathrm{~nm} \mathrm{rms}$ of residual aberrations (note that the overall budget for IRDIS optics between dichroic and detector is $36 \mathrm{~nm}$ rms which is in very good agreement with our measurements). This good result is due to

1. the exceptional quality of the SPHERE optics (all of them have been specified and delivered with less than a few nm rms) with specifc attention to the non-common ones.

2. the overall system alignment (and the days and weeks spent to make it both quasi perfect)

On the top of that, an optimized phase diversity algorithm has been applied to measure and correct for the very last residual aberrations. By doing that a SR of more than $99 \%$, that is $25 \mathrm{~nm} \mathrm{rms}$, is measured whereas the full iterative NCPA process of Phase Diversity measurements + reference slope modifications converges towards less than a few nm rms of residuals. The difference between the two numbers is essentially due to high spatial frequency aberrations which are neither sensed nor corrected by the NCPA loop but still presents in the image itself. Nevertheless, achieving more than $99 \%$ of SR on a 8 tons instruments with a volume of $6 \mathrm{mx} 4 \mathrm{mx} 2.5 \mathrm{~m}$ is already quite a performance and by far exceed the original specifications. A detailed description of the various wavefront sensor strategies in SPHERE could be found in [5]

\subsection{AO temporal behavior}

Temporal aspects are critical for XAO applications in order to improve the overall performance (better atmospheric correction as well as a better rejection of telescope and instrument vibrations). Nevertheless it requires significant effort both in terms of hardware (we need very fast component) and software (we need to be able to control them efficiently). In the SPHERE project, the two aspects have been carefully considered and several innovative solutions have been proposed. A detailed description of the SPHERE temporal behavior could be found in [6]. In a nutshell, the AO system has its expected temporal bandwidth of $70 \mathrm{~Hz}$ with a $1200 \mathrm{~Hz}$ frame rate and a $81 \mu$ s pure RTC latency. 


\subsection{The High Order deformable mirror issues}

This section is dedicated to the DM problems that have been identified as the highest risk item in the SPHERE system. It briefly describes the various DM issues as well as the mitigation procedures proposed by the consortium.

The HODM has been provided by the CILAS Company. It had to fulfill the following high specifications

- $41 \times 41$ actuator with a rectangular pitch $(4.5$ and $4.51 \mathrm{~mm})$ to match SPHERE optical design

- $1.5 \mu \mathrm{m}$ of mechanical interactuator stroke

- $10 \mu \mathrm{m}$ mechanical stroke with a shape at rest lower than $1 \mu \mathrm{m}$

- No dead actuator (and in particular no stuck actuator)

- High temporal response : $\mathrm{BW}>1 \mathrm{kHz}$ (no resonance below $5 \mathrm{kHz}$ ) for $A L L$ the actuators

- Operation conditions : up to $99 \%$ of humidity and $5^{\circ}-15^{\circ} \mathrm{C}$ (usual Paranal $T^{\circ}$ range)

Most of the specifications are not fulfill with the current DM (in italic). Although the system performance is barely

affected by the current BW limitation, the shape at rest (and especially its evolution with $\mathrm{T}^{\circ}$ ) and the DM dead actuators (from 2 when the DM has been delivered up to 17 at the moment this paper is written) represent the most severe limitations and medium / long term risks of the SPHERE system.

\subsubsection{The dead actuators: impact and mitigations}

The HODM dead actuators term gathers three classes of actuators: the ones with short-circuit, the ones with contact issues (between wires and piezo material) and the ones with extremely slow response (i.e. a very high resistance). All combined, they number has grown from 7 (at the very beginning of the AIT in 2010) to 17 (at the time we are written this paper). This increase is mainly due to DM operation in humidity condition larger than $50 \%$ and high $\mathrm{T}^{\circ}$. Therefore, an active dry system (Nitrogen laminar flow into the DM itself) allowing to reduce the humidity down to less than $50 \%$ has been added. Since then ( 9 months), there was no new dead actuators.

Considering the HODM design, any operation on these actuators is almost impossible and thus the system has to live with them. It has been demonstrated (both in simulation and in labs) that the impact on performance of such a number of dead (stuck to a 0 position) actuator is a killer from a contrast point of view.

Therefore, adopting a strategy proposed by the Gemini Planet Imager (GPI), modified Lyot stops (occulting the pupil area containing the dead actuators) have bene manufactured for EACH coronagraph (both in IR and VIS) of SPHERE.(see Figure 8). The gain in performance is demonstrated in Figure 8. With the new Lyot stop, ultimate performance is similar to a perfect DM (without any dead actuator).

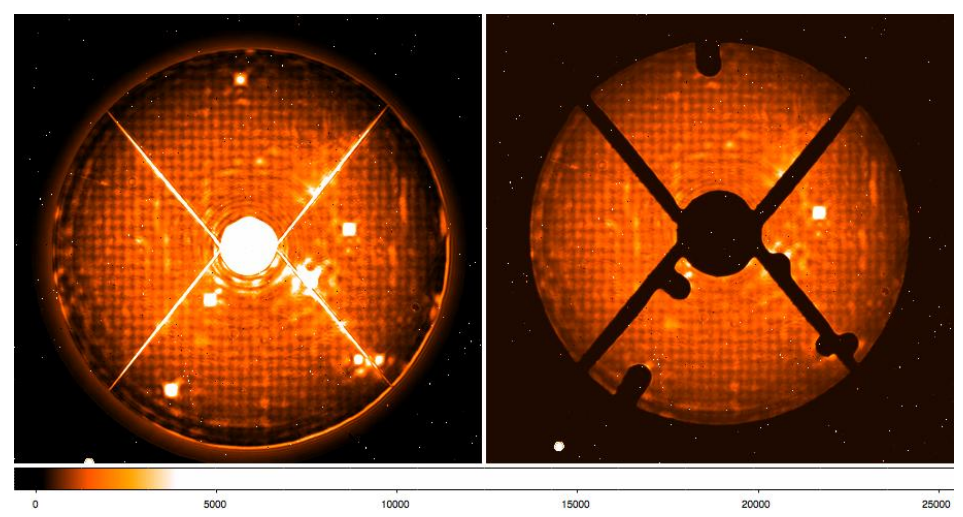

Figure 5 Pupil imaging showing the dead actuators and the new modified Lyot stop which masks them. Unfortunatly one additional actuator dies during the mask fabrication and is not properly covered. 


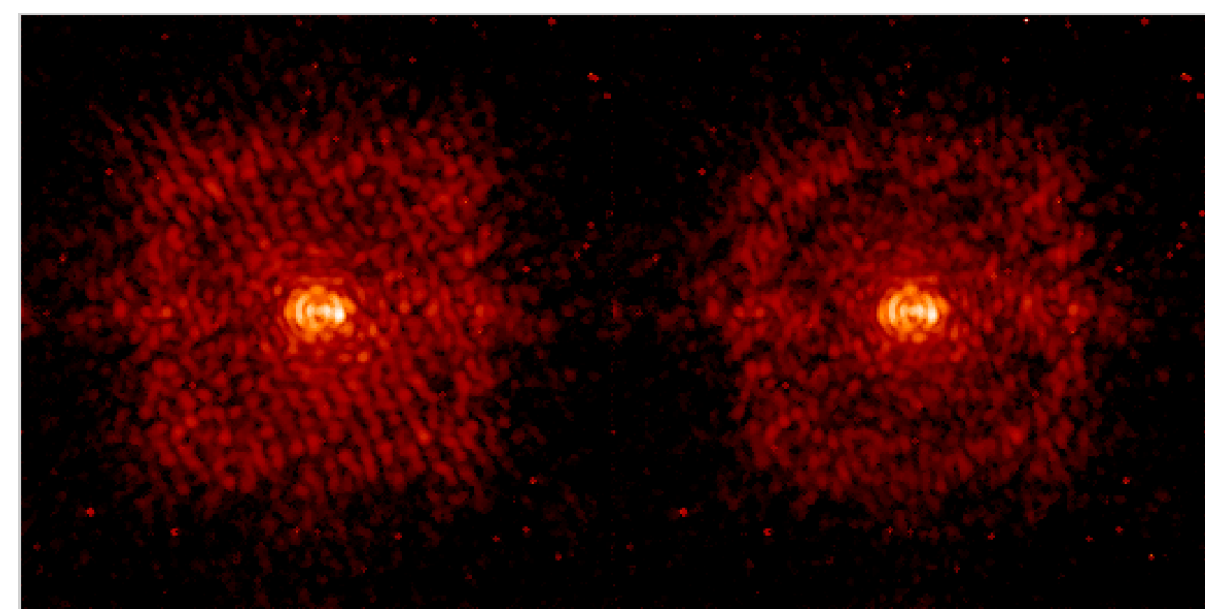

Figure 6 Coronagraphic image with "classical" Lyot stop [left] and with modified one [Right]. The gain in contrast is clearly visible despite the additional actuator which appears during the mask procurement.

\subsubsection{The shape at rest evolution with $\mathrm{T}^{\circ}$ : impact and mitigations}

The shape at rest is the initial shape of the DM before any deformation. It can be compensate by the DM itself but it reduce the DM stroke dedicated to the correction of turbulence itself. The specification was that less than $10 \%$ of the full DM stroke has to be dedicated to the DM flattening. Sadly, this specification is far from being reached. Not only has this value too high ( $50 \%$ in the best possible case) but it evolves with $\mathrm{T}^{\circ}$ (typical $1 \mu$ per degree) and can reached more than $100 \%$ of the full stroke when $\mathrm{T}^{\circ}$ exceed $18^{\circ} \mathrm{C}$ (see Figure 7). Nevertheless since the shape is mainly cylindrical, it is possible to compensate (at least partially) for it by introducing the opposite shape somewhere in the SPHERE common optical path. It has been done by modifying one of our Toric Mirror (the $3^{\text {rd }}$ one, so-called TM3) as explained hereafter.

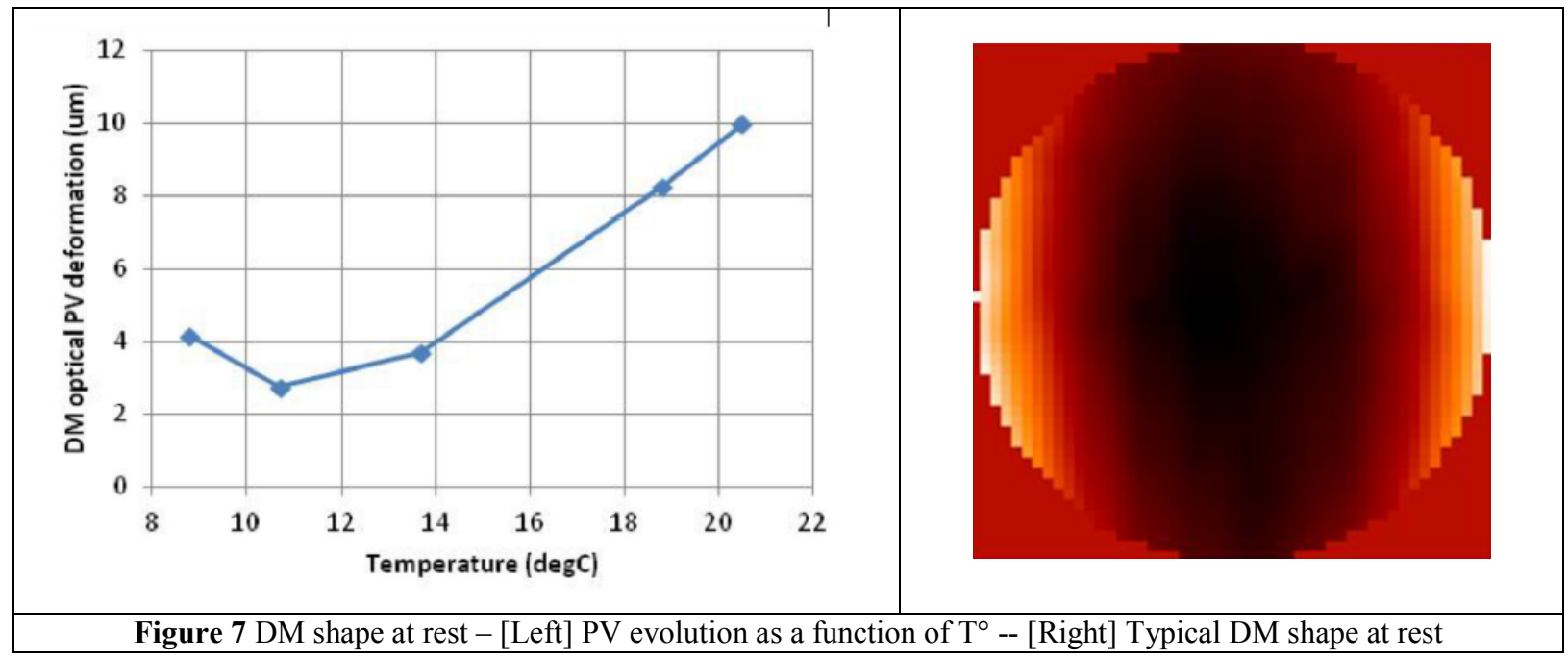

The basic idea was to install a warping harness on TM3 for cylinder compensation. A Shape optimisation has been made with FEA. Doing that it has been demonstrated that the influence function of $\mathbf{1}$ actuator corresponds to the required cylinder shape. The latest is therefore adjusted in real time during observations (see Figure 8).

This "woofer-tweeter" configuration allows us to work with our current HODM by ensuring that the final DM shape at rest is always lower than $2 \mu \mathrm{m}$ meca PV (in the extreme case) and a nominal case of less than $1 \mu \mathrm{m}$ meca PV which is less than $10 \%$ of the total DM stroke. 

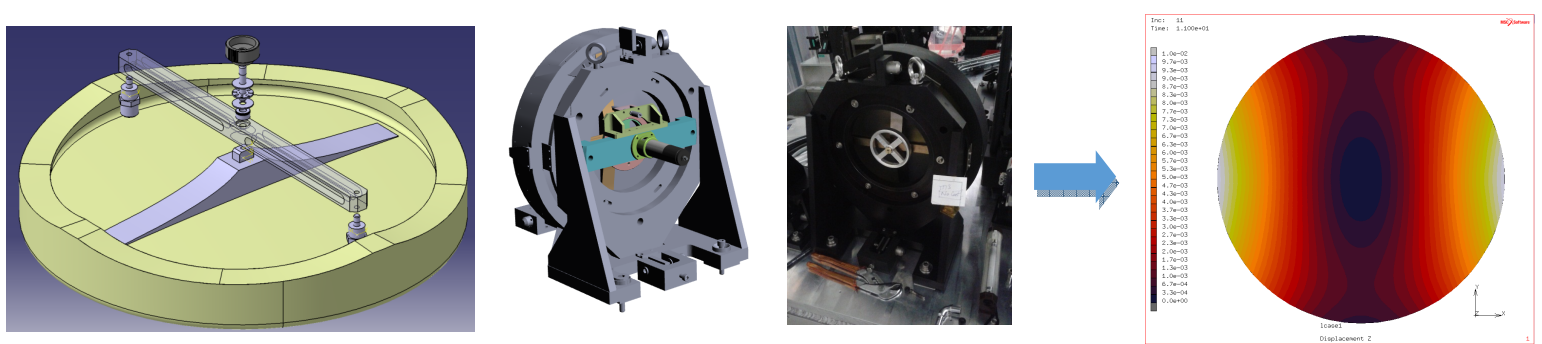

Figure 8 Scheme of active TM3, implementation and results.

Although the DM is not perfect yet, the current version allows a full SPHERE operation and does not significantly degrades the performance, its potential degradation remains a real concern for the project and one of the most importan risk for the whole SPHERE mission. CILAS, togather with ESO and TMT are currently working on a recovery plan to improve their manufacturing processes in order to have, for the future, a viable solution for the next DM generation.

\section{SAXO PERFORMANCE WITH TURBULENCE}

The previous section has demonstrated that, despite the DM issues) SAXO is achieving its required performance for nominal conditions (exceeding the goals in some aspects). We are now going to focus on performance in faint conditions.

\subsection{Nominal SAXO performance}

Let us now introduce turbulence. The SAXO nominal performance is define for the following conditions:

- Vmag 9, i.e. 100 photo-e- per sub-aperture and per frame on the WFS (@1.2 kHz)

- Seeing $=0.85 '$ and wind speed $=12.5 \mathrm{~m} / \mathrm{s}$

In these conditions, with a fully operational and optimized SAXO system (all loops closed, all optimization process "on" including Kalman Filtering on Tip Tilt) we have recorded classical PSFs and coronagraphic images on IRDIS (H2H3 mode). Direct imaging will give us Strehl ratio and will allow us quantify AO loop residuals in terms of $\mathrm{nm}$ rms. The coronagraphic image will provide the final performance in terms of detectivity.

From the classical PSF (see Figure 9), a SR has been computed: SR =90.3 2 \% @ 1589 nm (the error bars are estimated empirically from experience and knowledge of the system parameter accuracy). This SR can be translated into a global residual error: stotal $=81 \pm 10 \mathrm{~nm}$ rms

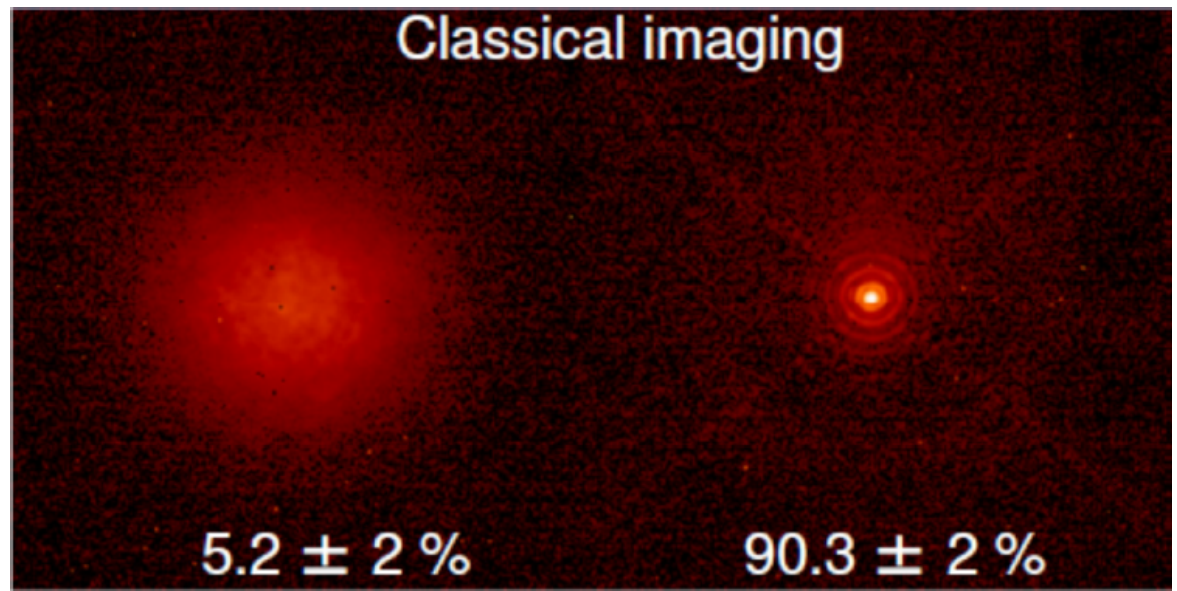

Figure 9 [Left] turbulent un-corrected image (seeing is estimated on the image to 0.85 ”), [Right] corrected image. 
Knowing that the fitting error for a 0.85 " seeing is estimated to sfit $=60 \pm 10 \mathrm{~nm}$ rms It leads to a residual rms on corrected modes (by the AO system) scorr $=54 \pm 14 \mathrm{~nm}$ rms which is well within the FDR specification and fully coherent with the simulation results SRsim $=89.8 \%$, leading to scorr,sim $=83 \mathrm{~nm} \mathrm{rms}$

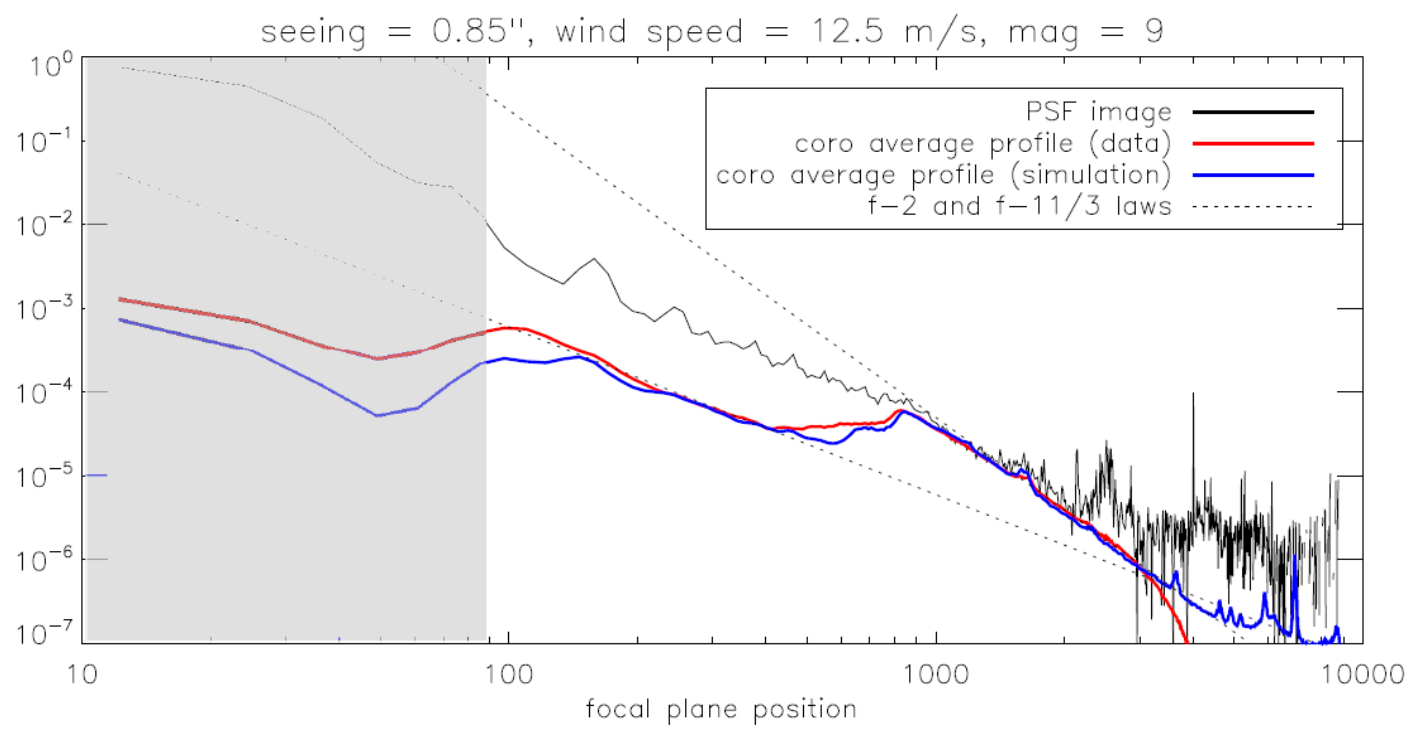

Figure 10 Raw coronagraphic images (circular average).

In red: experimental data, in blue: End2end simulations. Dotted line ( $\mathrm{f}^{-2}$ and $\mathrm{f}^{-11 / 3}$ laws)

Let us now analyze the raw coronagraphic images (Figure 10) and compare them with a full end2end simulation. The two profiles are remarkably similar. In the corrected area the profile follows a f-2 law signature of the noise and wind speed residual errors. After the AO cut-off frequency a f-11/3 law is found with the correct level (for 0.85 " seeing conditions). Only a very small over excitation is visible in the experimental data around 700 mas (less than a factor 1.5). It can be due to a WFS spatial filter a little bit larger in the real life than in simulation and to the dead actuators. In any case, the coronagraphic shape is very well mastered and understood. It follows the simulation data demonstrating that the AO loop is doing exactly what it supposes to do.

\subsection{SAXO performance in faint conditions}

Section 4.1 has demonstrated that, despite the DM issues) SAXO is achieving its required performance for nominal conditions (exceeding the goals in some aspects). We are now going to focus on performance in faint conditions.

Figure 11 show the evolution of SAXO performance (in terms of SR and PSF shape) for various SNR conditions. R magnitudes have been computed from flux measured on VIS-WFS sub-apertures and system measured transmissions. It shows that ultimate performance is achieved (in $\mathrm{H}$ band) for mag $<9$ and that the performance smoothly drops down to $15 \%$ for mag $>15.5$ which is a remarkable result. This is especially due to the very good system transmission and the exceptional performance of the WFS in terms of RON (<<1e-) and QE (deep depletion device). More detailed results (as well as first on-sky data) could be found in [5]. Karl-Schwarzschild-Str. 2

85748 Garching bei München

Germany 


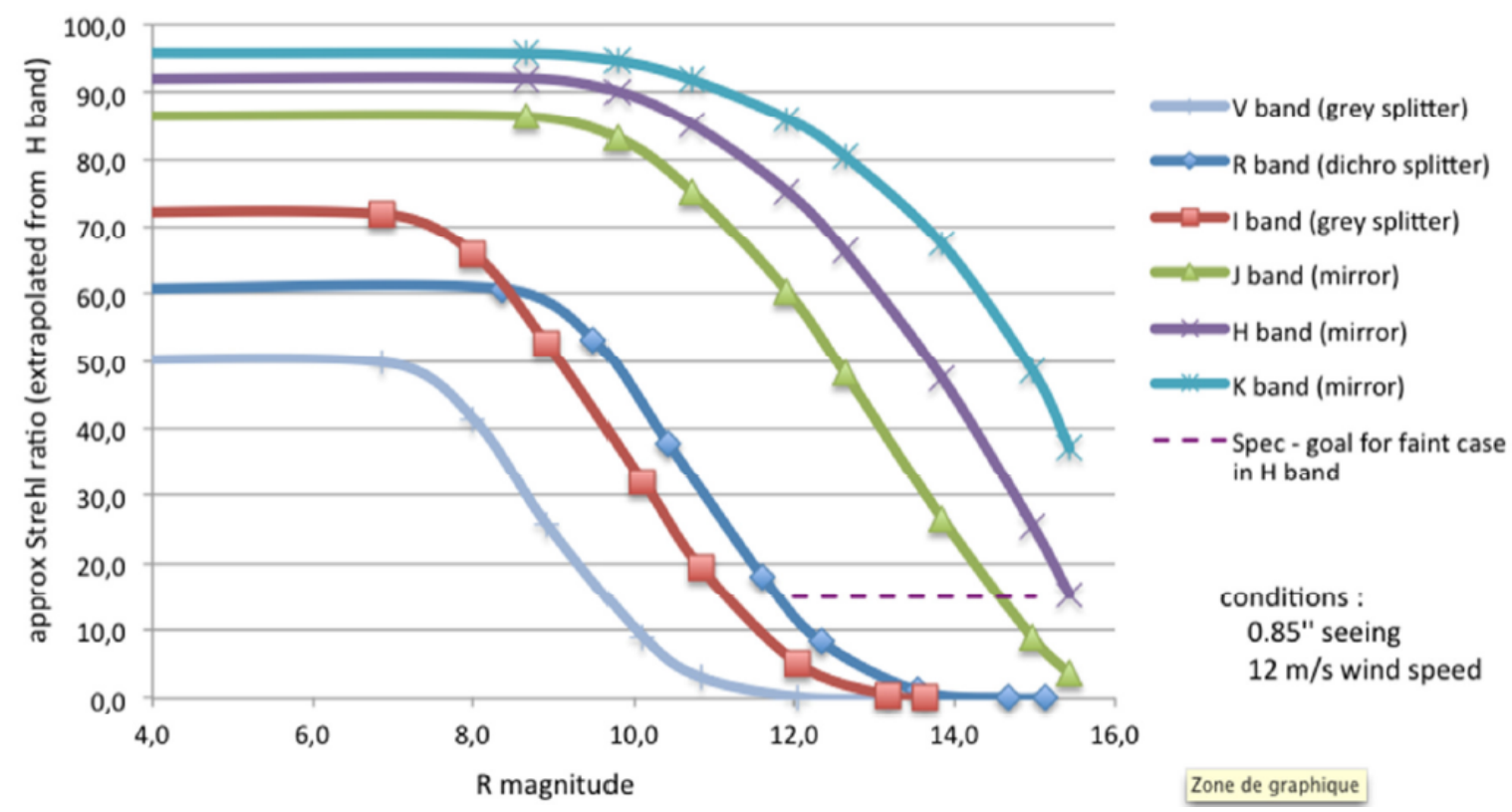

Figure 11 SR ratio as a function of GS magnitude (R band) for various SPHERE wavelengths (and thus SPHERE configuration in terms of beam-splitting between WFS and scientific paths)

\subsection{SAXO performance in poor (large seeing and wind speed) conditions}

In this section, the performance (as well as the robustness) of the system is studied in the poor condition regime, i.e. a seeing of 1.12 arcsec and two wind speed values of 12.5 and $30 \mathrm{~m} / \mathrm{s}$. These measurements have been performed in good $\mathrm{T}^{\circ}$ conditions $\left(<17^{\circ}\right)$ so that the DM had a relatively good shape at rest. First of all, despite some actuators in saturation (well handled by the anti-wind up and Garbage Collector processes), the loop was stable and robust during all the acquisition process (a few tens of minutes).

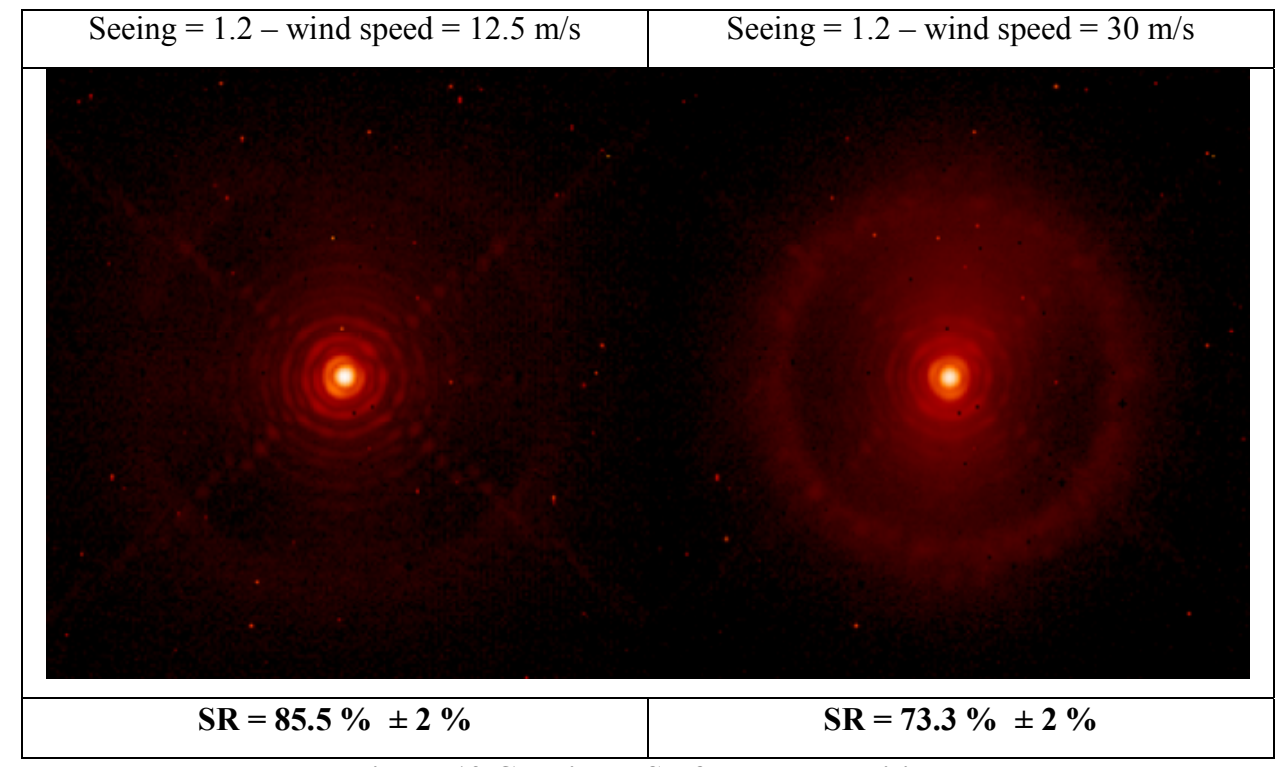

Figure 12 Classical PSF for poor conditions 


\section{FIRST ON-SKY RESULTS}

After two years of intensive test in labs at Grenoble, the SPHERE instrument and its SAXO AO system has been shipped to Paranal Observatory in February 2014, reintegrated and fully retested in the VLT integration hall (in March and April 2014) on the bottom of the mountain and finally installed on the telescope the ten last days of April. SPHERE has seen its first on-sky photon the $4^{\text {th }}$ of May and the AO loop has been closed less than 20 min after the beginning of operation. The three first week of May have been dedicated to functional tests and very first performance analysis. We present here the very first results obtained on-sky, knowing that, they are deduced from the very first data during the early days of the instrument commissioning. Exhaustive analysis and full performance assessment will be provided in the coming months after the various SPHERE commissioning run (from July to October 2014).

\subsection{Classical images}

Let start with classical PSF images (without coronagraph).

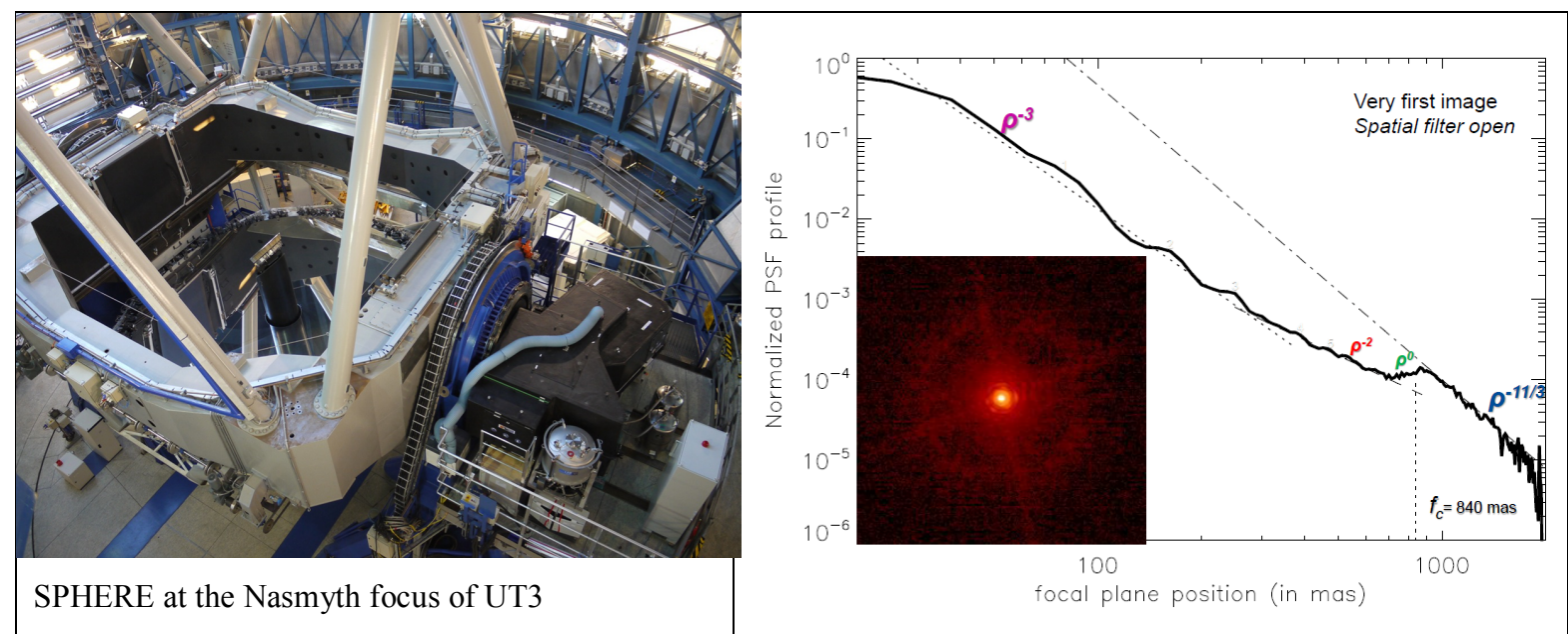

Figure 13 [Left] Nice photo of SPHERE on UT3 - [Right] The first SPHERE Hband PSF. Image and circular profile. The AO spatial cut off frequency is clearly visible at 840 mas (as expected). Several regime (see text for details) have been identified on the image.

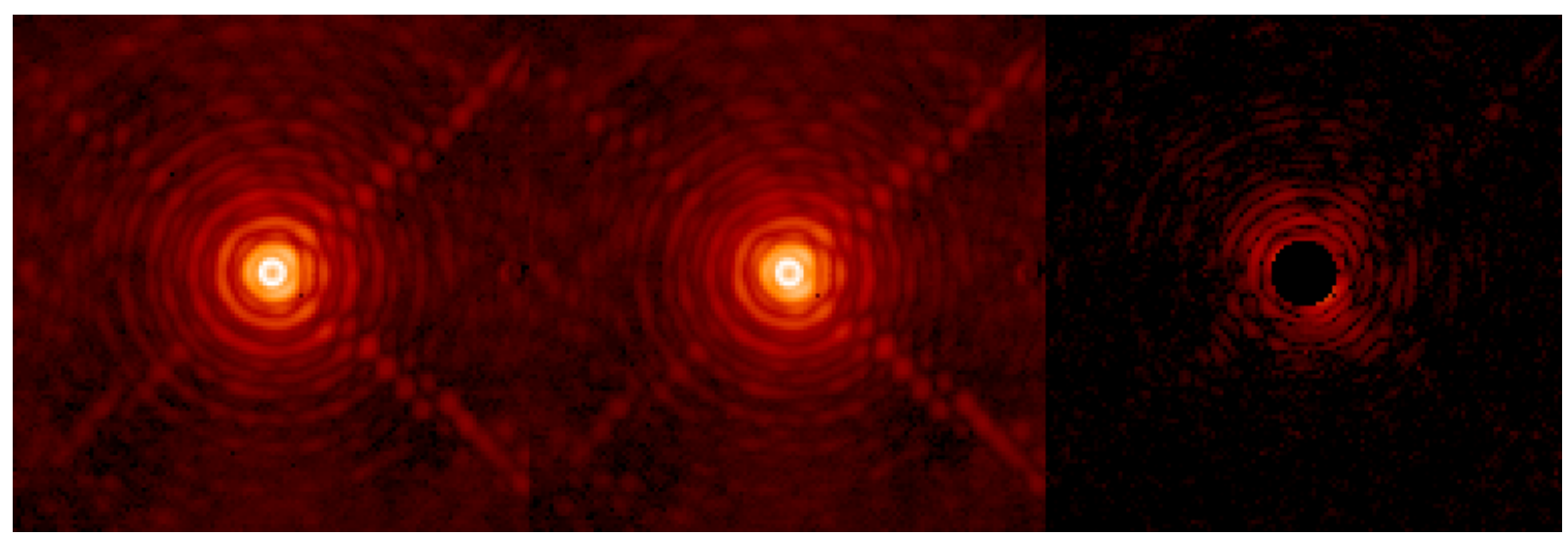

Figure 14 Illustration of image and coronagraphic PSF stability. From Left to Right. Two saturated images acquired with a 20 min interval. Intensity difference (absolute value). All the data are plotted in log scale

The very first PSF data acquired on SPHERE (on H band) shows very nice behavior with a clear diffraction limited area, a very well defined AO curt-off frequency at its expected location (840 mas in radius in $\mathrm{H}$ band). The various regimes are illustrated in Figure 13. 
A very first analysis of the PSF stability has been made on 20 min non-coronagraphic images (illustrated inFigure 14). An extremely good stability of the AO correction as well as of the residual "static" speckle is demonstrated. The SR for these data (obtained on an unsaturated PSF) is $83 \%$ for a seeing of 0.7 arcsec.

\subsection{Coronagraphic images}

Even though classical PSF are interesting for a very first analysis of the SAXO loop behavior, the main SPHERE mode is coronagraphy. Removing the diffraction pattern with a coronagraph allows us to go deeper into the fine analysis and understanding of the SAXO performance and stability.

Let us first illustrate the coronagraphic mode with 3 beautiful images (see Figure 15) obtained at 3 wavelengths $(\mathrm{H}$, J and $\mathrm{Y}$ in nominal SAXO operation (meaning that all the AO feature were applied: Kalman filtering on TT, modal gain optimization, anti-wind up and garbage collection, spatial filter closed at its optimal value, IR loop engage for fine centering on the coronagraph and pupil stabilization). The expected speckle evolution with wavelengths (as well as the size of the corrected area) is clearly visible on the data. The cleaning of the corrected area (and in particular the very strong dark region) is particularly impressive. The spatially filtered SH impact is essential here. We will come back on this specific SAXO feature in the next section.

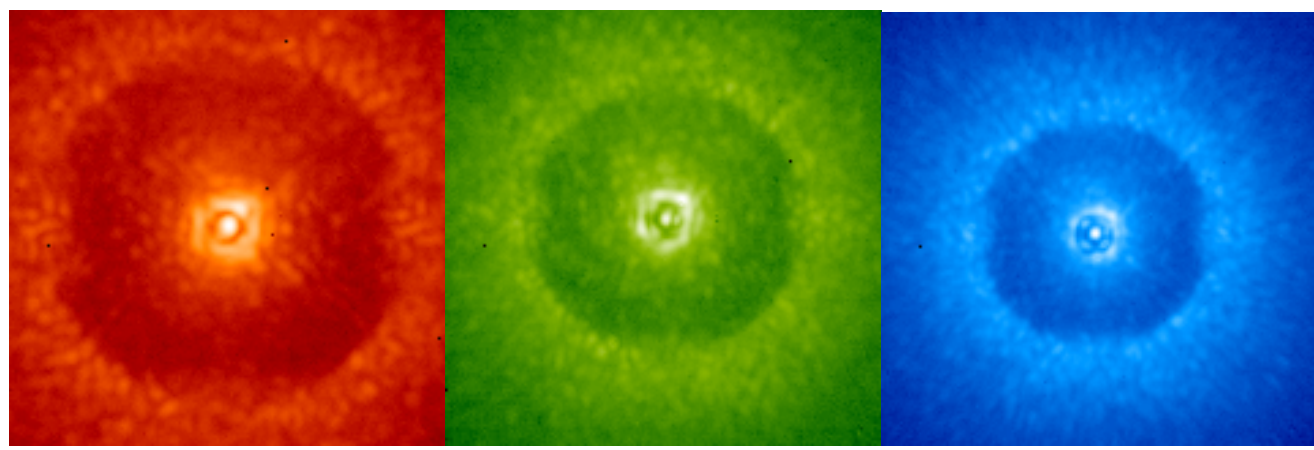

Figure 15 Coronagraphic images obtained at 3 wavelengths (from left to right : H, J and Y bands). Classical images have also been acquired and SR for each band is estimated to 80,70 and $60 \%$ respectively. Seeing has been estimated to 0.7 "

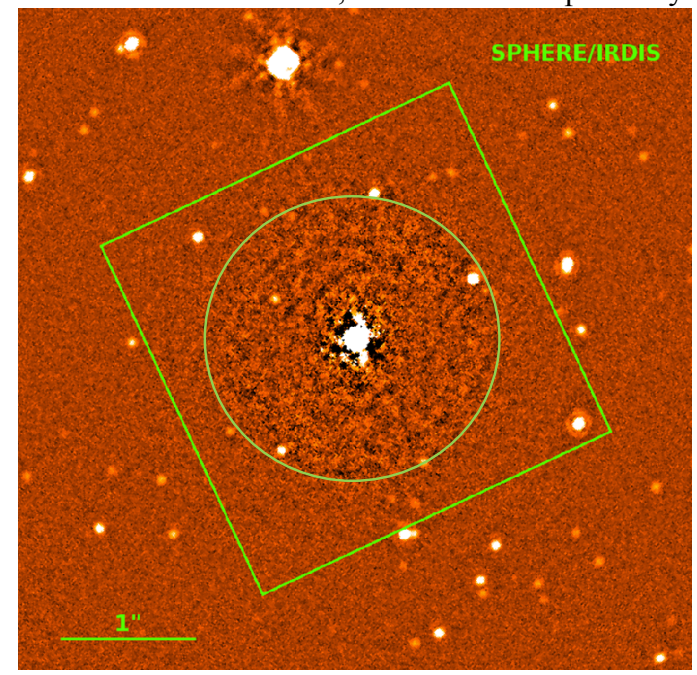

Figure 16 Coronagraphic and ADI observation of HR165054. Full observation $=20$ min

Observing an astrometric field in ADI mode (with pupil stabilization) has allowed us to demonstratre that nonatmospheric speckle (static) are really stable and can be accurately removed by the ADI procedure. The AO corrected area is shown with the green circle. Focal plane residue does not show any speckle like features. 


\subsection{Impact of the SH spatial filter}

Now, lets go back to the spatially filtered SH. The goal of this device is to remove the aliasing effects in the WFS measurements [7], [8]. Figure 17 shows the very nice behavior of coronagraphic images (i.e. the increase of AO correction and ultimate performance) as a function of the spatial filter size. The gain brought by the spatially filtered SH is up to a factor 3. We have managed to go down to $1.1 \lambda / \mathrm{d}$ (d being the sub-aperture size) which is close to the theoretical limit and which allows us to have a real aliasing free WFS. A detailed analysis and comparison with simulation results can be found in [5].
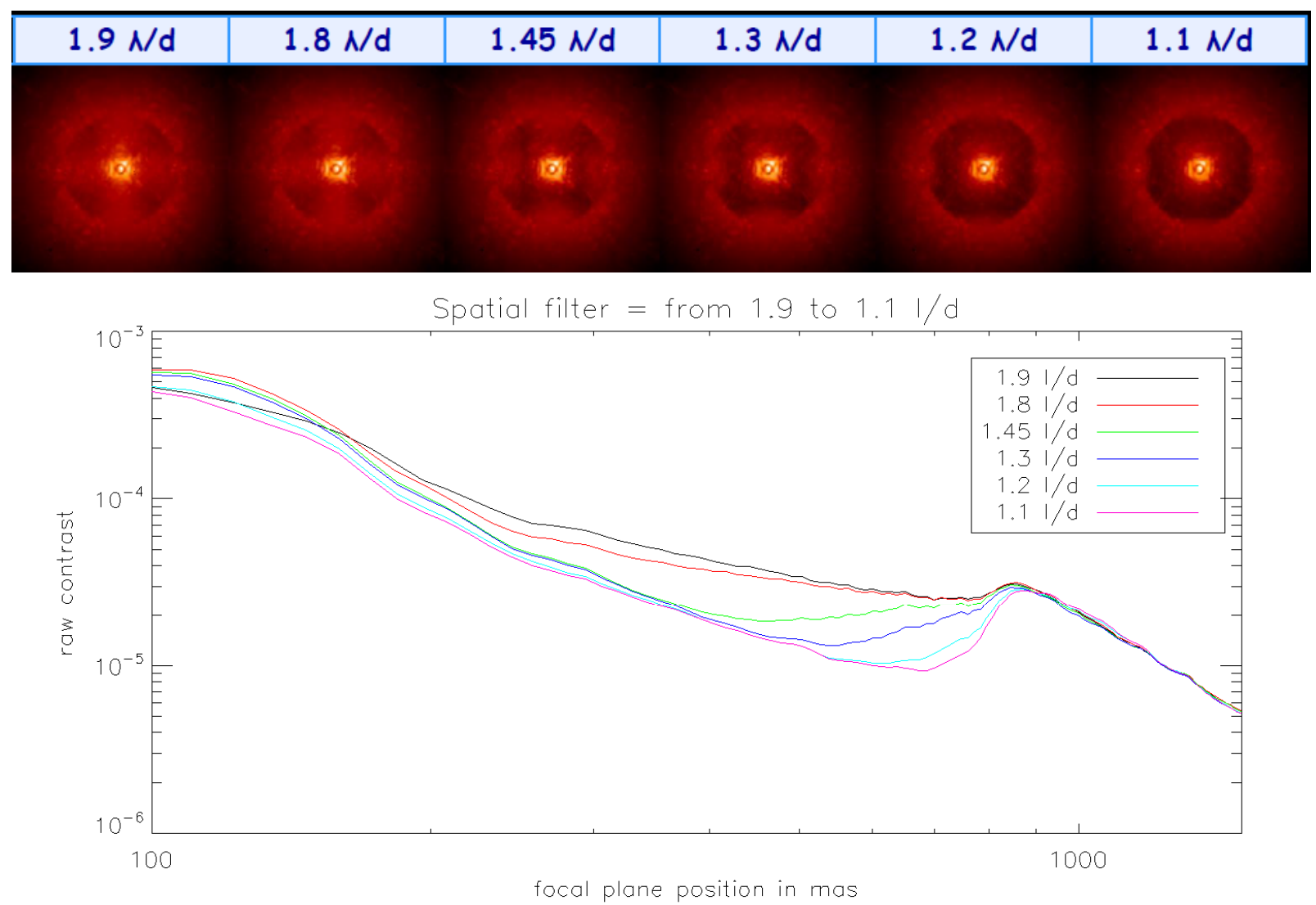

Figure 17 Evolution of the coronagraphic images as a function of the spatial filter size. Up : images, Down : coronagraphic profiles

\section{SOME NICE IMAGES ... AND MORE TO COME}

A detailed description of SPHERE overall performance (in terms of final contrasts and detectivity aspects) will be found in [4]. In this section we only show some nice images obtained during the first commissioning. The have been post processing (either using ADI or deconvolution processes). The final results show the very good AO efficiency and, more importantly, its extremely good stability with time. 


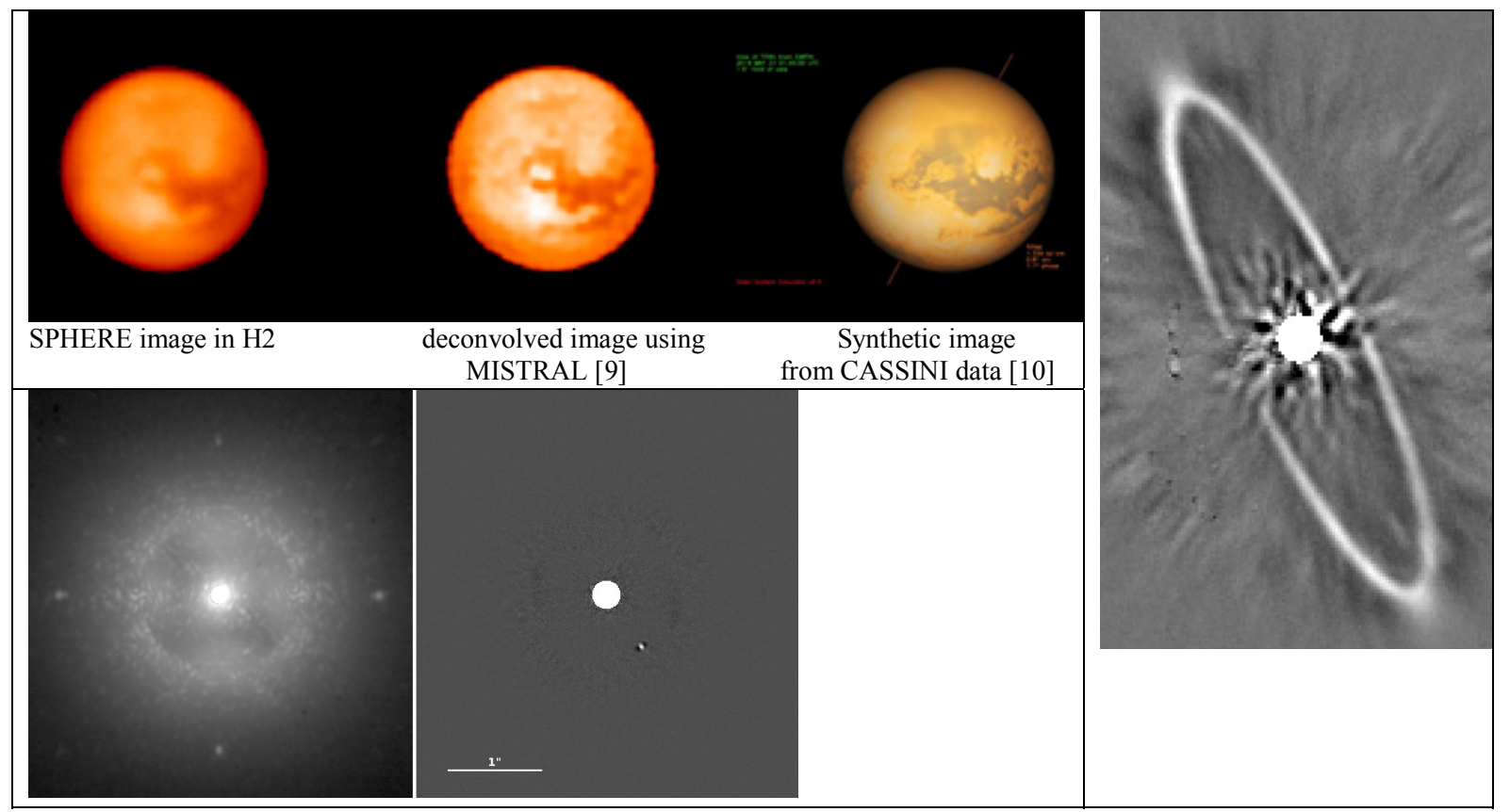

Figure 18 some nice SPHERE images

\section{CONCLUSION}

SPHERE-SAXO is 12 years of project involving 12 institute in Europe and more than 250 Full Time Equivalents. The system design has barely evolved from the early sketches (in 2002) to the final telescope implementation (2013-14). At the end, the AO system gathers state of the art components (detectors, deformable mirror and real time computer) and some of its innovative features allow to have performance which has exceeded the original specifications. Even though we have experimented some problems with our high order deformable mirror, solutions have been proposed by the consortium to mitigate these effects and to be able to work with the current device. Nevertheless, DM remains, for the moment, the main risk of failure for the whole system. Back-up, long-term, solutions are now under investigation between ESO, CILAS and the SPHERE consortium to deal with this particular point.

Extensive laboratory tests have fully demonstrated both the performance, the reliability and the stability of the AO loops (and of SAXO and SPHERE as a whole).

The first on-sky commissioning has confirmed laboratory tests by providing unprecedented images and coronagraphic data on the VLT. The system stability has been re-demonstrated on-sky and the first performance assessment, even though Strehl ratio is slightly smaller than expected due to bad weather conditions and residual high frequencies on the telescope M2, are more than encouraging. The principal feature of SAXO have already been successfully tested from a functional point of view and fine tuning of the AO system as well as of the telescope control should allow to reach, in the coming months, the full system performance.

\section{REFERENCES}

[1] .J-L. Beuzit, D. Mouillet, C. Moutou, K. Dohlen, P. Puget, T. Fusco, and A. Boccaletti, "A planet finder instrument for the VLT," in Proceedings of IAU Colloquium 200, Direct Imaging of Exoplanets: Science \& Techniques (Cambridge University Press, 2005), pp. 317-323

[2] T. Fusco et al "High-order adaptive optics requirements for direct detection of extrasolar planets: Application to the SPHERE instrument” Optics Express, Vol. 14, Issue 17, pp. 7515-7534 (2006)

[3] J-F Sauvage J.-F., Fusco T., Rousset G., Petit C., "Calibration and Pre-Compensation of Non-Common Path Aberrations for eXtreme Adaptive Optics”, JOSA A, Vol. 24, Issue 8, pp. 2334-2346 (2007) 
[4] J.-L. Beuzit et al, "SPHERE: a planet finder instrument for the VLT", paper 9148-17, this proceeding

[5] J.-F; Sauvage et al "Wave-front sensor strategies for SPHERE: first on-sky results and future improvements", paper 9148-155, this proceeding

[6] C. Petit et al "SPHERE eXtreme AO control scheme: final performance assessment and on sky validation of the first auto-tuned LQG based operational system", Paper 9148-23, this proceeding

[7] L.A.Poyneer and B. Macintosh, "Spatially filtered wave-front sensor for high-order adaptive optics", JOSA A, Vol. 21, Issue 5, pp. 810-819 (2004)

[8] T. Fusco et al "Closed-loop experimental validation of the spatially filtered Shack-Hartmann concept", Optics Letters, Vol. 30, Issue 11, pp. 1255-1257 (2005)

[9] L. Mugnier, T Fusco, and J-M Conan, "MISTRAL: a myopic edge-preserving image restoration method, with application to astronomical adaptive-optics-corrected long-exposure images”, JOSA A, Vol. 21, Issue 10, pp. 1841-1854 (2004)

[10]: http://space.jpl.nasa.gov/ 